\title{
Background-Suppressed MR Venography of the Brain Using Magnitude Data: A High-Pass Filtering Approach
}

\author{
Zhaoyang Jin, ${ }^{1,2,3}$ Ling Xia, ${ }^{1,2}$ Minming Zhang, ${ }^{4}$ and Yiping P. Du ${ }^{1,2}$ \\ ${ }^{1}$ Department of Biomedical Engineering, Zhejiang University, Hangzhou 310027, China \\ ${ }^{2}$ Key Laboratory for Biomedical Engineering of Education Ministry of China, Hangzhou, China \\ ${ }^{3}$ College of Automation, Hangzhou Dianzi University, Hangzhou, China \\ ${ }^{4}$ Department of Radiology, School of Medicine, Second Affiliated Hospital, Zhejiang University, Hangzhou, China
}

Correspondence should be addressed to Yiping P. Du; yipingdu@zju.edu.cn

Received 24 October 2013; Revised 10 February 2014; Accepted 14 February 2014; Published 10 June 2014

Academic Editor: Yuanjie Zheng

Copyright (C) 2014 Zhaoyang Jin et al. This is an open access article distributed under the Creative Commons Attribution License, which permits unrestricted use, distribution, and reproduction in any medium, provided the original work is properly cited.

\begin{abstract}
Conventional susceptibility-weighted imaging (SWI) uses both phase and magnitude data for the enhancement of venous vasculature and, thus, is subject to signal loss in regions with severe field inhomogeneity and in the peripheral regions of the brain in the minimum-intensity projection. The purpose of this study is to enhance the visibility of the venous vasculature and reduce the artifacts in the venography by suppressing the background signal in postprocessing. A high-pass filter with an inverted Hamming window or an inverted Fermi window was applied to the Fourier domain of the magnitude images to enhance the visibility of the venous vasculature in the brain after data acquisition. The high-pass filtering approach has the advantages of enhancing the visibility of small veins, diminishing the off-resonance artifact, reducing signal loss in the peripheral regions of the brain in projection, and nearly completely suppressing the background signal. The proposed postprocessing technique is effective for the visualization of small venous vasculature using the magnitude data alone.
\end{abstract}

\section{Introduction}

MR venography (MRV) has demonstrated substantial clinical significance in the diagnosis of intracranial vascular diseases, such as venous thrombosis and arteriovenous malformations [1-3]. Flow-sensitive 2D time-of-flight and phase-contrast techniques have been commonly used for intracranial MRV [1-3]. MRV with administration of contrast media has been used for the visualization of dural sinuses and small veins with low blood flow and diagnoses of cerebral vasoocclusive diseases [4-7]. MR susceptibility-weighted imaging (SWI) has been demonstrated to be an excellent technique in the visualization of the cerebral venous vasculature without exogenous contrast media [8-11]. The susceptibility difference between deoxygenated hemoglobin in venous blood and brain parenchyma and the field inhomogeneity within the surrounding tissue around the veins result in partial volume signal cancellation in a voxel and generate a phase shift in the voxel $[12,13]$. SWI data have usually been acquired using a 3D spoiled gradient-echo (SPGR) pulse sequence with a relatively long echo time (TE). In conventional SWI processing, both signal reduction and phase shift are used for the display of venous vasculature. Excellent venous contrast can be obtained by combining the high-pass filtered phase data with the magnitude data.

Current SWI methods either use a homodyne filtering algorithm or a combination of phase unwrapping and highpass filtering $[14,15]$. These methods, however, are subject to the off-resonance artifact in regions with severe field inhomogeneity because of the incomplete suppression of background phase in the high-pass filtered phase images in these regions $[8,10,16,17]$. This artifact can be reduced by increasing the filter size in both methods [14-17], which may have the drawback of reducing the contrast of small veins [17]. Signal loss is also commonly observed in the peripheral regions of the brain in the minimum-intensity projection (mIP) of the 3D SWI data [17]. In addition, the contrast of veins in the high-pass filtered phase images is highly dependent on the orientation of the veins relative to the main field, voxel size, and voxel aspect ratio $[12,13]$. 
Several techniques have been developed to reduce the offresonance artifact in regions with severe field inhomogeneity in SWI [17]. These techniques include phase unwrapping $[14,15]$, a regional suppression of phase artifact using local field gradient mapping [17], and the use of a susceptibility model [18]. Signal loss in the peripheral region of the brain was reduced by the use of tissue-air volume segmentation algorithms [17, 19-21]. Despite the effectiveness of these techniques, residual image artifacts often remain in SWI.

In this study, we present a background-suppressed MRV (BS-MRV) technique for the visualization of the cerebral venous vasculature using 3D SPGR magnitude data acquired without exogenous contrast media. In this technique, visualization of the venous vasculature in the magnitude data is enhanced by background suppression through postprocessing. This technique has a major advantage of simplicity since only the magnitude data are used. This eliminates the need for phase data and the possible technical challenges in dealing with phase errors and phase unwrapping. The BS-MRV technique has the advantages of (1) the enhanced visibility of small veins, (2) diminished off-resonance artifacts, (3) reduced signal loss in the peripheral regions of the brain in the MIP display, and (4) a nearly completely suppressed background signal.

\section{Materials and Methods}

2.1. Background Suppression Using a High-Pass Filter. In this study, a high-pass (HP) filtering technique was applied in the Fourier domain of the magnitude data to enhance the signal of venous vasculature through background suppression. The implementation of the BS-MRV technique includes the following steps. (1) Form a 3D complex image dataset with the real component filled with the magnitude image data and the imaginary component filled with zeroes. (2) Apply Fourier transform of the 3D complex data. (3) Apply a 2D HP filter to the $k_{x}-k_{y}$ plane for each partition of the 3D data for background suppression, where $k_{x}$ is the readout direction and $k_{y}$ is the phase encoding direction. Because a 2D HP filter is applied to the phase data along the in-plane directions in conventional SWI, we decided to use the same 2D HP filtering approach in this study. (4) Apply an inverse Fourier transform to obtain a $3 \mathrm{D}$ complex dataset in the image domain. (5) Take the real component of the 3D complex dataset to form a 3D HP-filtered dataset, $I_{\mathrm{HP}}(x, y, z)$. Two types of HP filters were studied for background suppression in Step 3, namely, an inverted Hamming $(\mathrm{iH})$ and an inverted Fermi (iF) filter. The iH filter, with a full size of $2 H_{x}$ and $2 H_{y}$, was applied at the center of $k$-space:

$$
\begin{aligned}
& \mathrm{iH}\left(n_{x}, n_{y}\right) \\
& 0.46\left[1.0-\cos \left(\pi \sqrt{\frac{n_{x}^{2}}{H_{x}^{2}}+\frac{n_{y}^{2}}{H_{y}^{2}}}\right)\right], \\
& \text { for } \frac{n_{x}^{2}}{H_{x}^{2}}+\frac{n_{y}^{2}}{H_{y}^{2}} \leq 1, \\
& \text { elsewhere. }
\end{aligned}
$$

The iF filter for square FOV $\left(N_{x}=N_{y}\right)$ is given by

$$
\begin{aligned}
& \operatorname{iF}\left(n_{x}, n_{y}\right) \\
& =\left\{\begin{array}{c}
1-\frac{1}{\left[1+\exp \left(\sqrt{n_{x}^{2}+n_{y}^{2}}-R\right) / W\right]}, \\
\text { for } \sqrt{n_{x}^{2}+n_{y}^{2}} \leq R+W, \\
\text { elsewhere, }
\end{array}\right.
\end{aligned}
$$

where $2 R$ is the size of the filter (i.e., $R$ is the radius of the filter) and $W$ is the width of the transition. When $N_{x} \neq N_{y}$, $2 R_{x}$ and $2 R_{y}$ are the size of the filter along the $k_{x}$ and $k_{y}$ directions, respectively. An iF filter is expected to have more effective background suppression than an $\mathrm{iH}$ filter with the same size because the former one has a broader stopband than the latter one. Another Fermi filter was applied to improve SNR and reduce the angular dependency of spatial resolution by suppressing the data on the corners of the rectangular region in $k$-space [22]. Zero-filled interpolation was applied along both $k_{x}$ and $k_{y}$ directions to improve the smoothness of the vessel contrast by reducing the partial volume effect.

2.2. Scaling and Display of BS-MRV Data. The background signal in the $I_{\mathrm{HP}}(x, y, z)$ images is expected to be nearly completely suppressed with intensity close to zero, while the venous vasculature is expected to have negative values because veins have a negative contrast in the original magnitude data. The contrast of the veins in the $I_{\mathrm{HP}}(x, y, z)$ data, however, is dependent on the size of the HP filter. Using a larger filter size would result in reduced vascular signal because of the greater signal suppression near the central region of $k$-space. In the conventional display of the MRV data, the brightness and contrast of the veins are affected by the voxels with signal at the high and low ends of the intensity range of the data. Instead of using the intensity range of the data, the standard deviation (STD), $\sigma$, of the HP-filtered data, $I_{\mathrm{HP}}(x, y, z)$, was used to scale the data, so that the brightness and contrast of the veins have substantially reduced dependency on the voxels with intensity near the maximum and minimum in the data. On the other hand, the brightness of the background is affected by the mean intensity, $I_{m}$, in $I_{\mathrm{HP}}(x, y, z)$. For this reason, $I_{m}$ was subtracted from $I_{\mathrm{HP}}(x, y, z)$ before the scaling to minimize the dependency of the brightness in background on filter size. The calculations of $\sigma$ and $I_{m}$ were repeated twice based on the histogram of $I_{\mathrm{HP}}(x, y, z)$ in a selected region of interest in the brain. The initial calculations of $\sigma$ and $I_{m}$ were used to exclude the voxels with the low intensity $<I_{m}-3 \sigma$ and high intensity $>I_{m}+3 \sigma$ for improved estimation of STD and mean in the refined calculation. 
TABLE 1: The standard deviation and mean intensity of the 3D BR-MRV datasets obtained with the inverted Hamming and Fermi filters $\left(\times 10^{-5}\right)$. The maximum intensity of the original 3D magnitude dataset prior to the filtering was normalized to 1.0.

\begin{tabular}{lcccccccccccc}
\hline \multirow{2}{*}{ Filter size } & \multicolumn{1}{c}{ Inverted Hamming } & \multicolumn{4}{c}{ Inverted Fermi } \\
& $\sigma_{t}$ & $\sigma_{a}$ & $\sigma$ & $I_{m, t}$ & $I_{m, a}$ & $I_{m}$ & $\sigma_{t}$ & $\sigma_{a}$ & $\sigma$ & $I_{m, t}$ & $I_{m, a}$ & $I_{m}$ \\
\hline $12 \times 9$ & 247 & 68.5 & 260 & 147 & 1.6 & 24.2 & 237 & 68.6 & 225 & 30.8 & -6.3 & -13.4 \\
$16 \times 12$ & 240 & 68.5 & 241 & 98 & -1.8 & 7.8 & 229 & 68.3 & 207 & 21.5 & -6.6 & -13.4 \\
$24 \times 18$ & 227 & 68.5 & 216 & 56 & -4.4 & - & 210 & 68.4 & 182 & 9.2 & -6.5 & -11.6 \\
$32 \times 24$ & 216 & 68.2 & 198 & 37 & -5.4 & - & 196 & 68.0 & 169 & 3.1 & -6.5 & -10.0 \\
$48 \times 36$ & 200 & 67.6 & 176 & 19 & -6.2 & - & 181 & 66.9 & 153 & -1.0 & -6.4 & -8.2 \\
$64 \times 48$ & 189 & 67.3 & 163 & 11 & -6.2 & - & 171 & 64.7 & 141 & -2.3 & -6.7 & -7.1 \\
\hline
\end{tabular}

$\sigma_{t}$ : standard deviation of intensity in brain tissue; $\sigma_{a}$ : standard deviation of intensity in air; $\sigma$ : standard deviation of intensity in the entire dataset; $I_{m, t}:$ mean of intensity in brain tissue; $I_{m, a}$ : mean of intensity in air; $I_{m}$ : mean of intensity in the entire dataset.

The BS-MRV data, $I_{\mathrm{BS}-\mathrm{MRV}}(x, y, z)$, was generated using the following linear scaling procedure to reduce the dependency of the display vessel contrast on filter size:

$$
\begin{gathered}
I_{\mathrm{BS}-\mathrm{MRV}}(x, y, z)=\frac{\left[I_{\mathrm{HP}}(x, y)-I_{m}\right]}{\sigma}, \\
\text { if }-\eta \sigma<\left[I_{\mathrm{HP}}(x, y, z)-I_{m}\right]<0, \\
I_{\mathrm{BS}-\mathrm{MRV}}(x, y, z)=-\eta, \quad \text { if }\left[I_{\mathrm{HP}}(x, y, z)-I_{m}\right]<-\eta \sigma, \\
I_{\mathrm{BS}-\mathrm{MRV}}(x, y, z)=0, \quad \text { if }\left[I_{\mathrm{HP}}(x, y, z)-I_{m}\right]>0 .
\end{gathered}
$$

The venous vasculature has a negative contrast in $I_{\mathrm{BS}-\mathrm{MRV}}(x, y, z)$ with an intensity range from $-\eta$ to 0 .

2.3. Data Acquisition. The MRV data were acquired on a GE 3T scanner (Milwaukee, WI, USA) in a volume slightly above the circle of Willis. Studies were performed on six healthy subjects who provided written informed consent approved by the local Institutional Review Board. High-order shim was applied prior to the scans to improve the overall field inhomogeneity.

The first dataset, with a size of $512 \times 384 \times 64$, was acquired along the transverse direction using a 3D SPGR pulse sequence with a birdcage head coil. The scan parameters were field of view $($ FOV $)=26 \times 19.5 \mathrm{~cm}^{2}, 1.0 \mathrm{~mm}$ slice thickness, full echo acquisition, readout bandwidth $=$ $\pm 31.3 \mathrm{kHz}$, and TE/TR $/ \alpha=20 \mathrm{~ms} / 34 \mathrm{~ms} / 20^{\circ}$. The frequency encoding direction had a FOV of $26 \mathrm{~cm}$ with 512 sampling points. The scan time was 14.8 minutes. Flow compensation was applied along the readout direction to reduce the phase variation caused by blood flow. The $3 \mathrm{D}$ complex SWI data were reconstructed offline using MATLAB (The MathWorks, Inc., Natick, MA, USA).

The second dataset was acquired with five subjects using an eight-channel phased-array RF coil with the same scan parameters as in the first dataset, except for the use of a smaller flip angle of 12 degrees for reduced partial T1 saturation of signal in cerebrospinal fluid (CSF). The magnitude data acquired from each of the coils was combined to form a composite dataset using the square root of the sum of squares.

The third dataset was acquired at a lower resolution, with a size of $384 \times 312 \times 32$, using a 4 -echo MR angiography and venography pulse sequence [23] with the same birdcage coil. The MRA data were acquired at the first echo and three volumes of MRV data were acquired at the second, third, and fourth echoes. The FOV was $20 \times 16 \mathrm{~cm}^{2}$ and the slice thickness was $1.6 \mathrm{~mm}$. All four echoes were acquired with a $66.25 \%$ partial echo and a flip angle of 20 degrees. The partial echo data were acquired in a readout window of $8.14 \mathrm{~ms}$ with a bandwidth of $\pm 15.6 \mathrm{kHz}$. The TEs of these four echoes were TE1/TE2/TE3/TE4 = 5.5/19.7/24.8/39.0 ms. The TR was $47 \mathrm{~ms}$ and the scan time was 7 minutes, 53 seconds. Flow compensation was applied along the readout and in-plane phase-encoding directions to the first echo to reduce flow artifact. The partial-echo datasets were reconstructed offline using the POCS [24] algorithm.

\section{Results}

The mean and STD of the HP-filtered 3D images are shown in Table 1 . These images were obtained by applying the $\mathrm{iH}$ and $\mathrm{iF}$ filters with different filter sizes to the 3 D SPGR $(512 \times 384 \times 64$, zero-filled to $1024 \times 768 \times 128)$ dataset. The following was observed. (1) The intensity of the filtered datasets had tight distributions with small STDs. For example, the overall STD of the entire 3D imaging volume in the filtered dataset $(\sigma)$ with iH $32 \times 24\left(2 H_{x}=32,2 H_{y}=24\right)$ was less than $0.2 \%$ of the maximum intensity of the original dataset. (2) The STDs in air, $\sigma_{a}$, were about one-third smaller than that in tissue, $\sigma_{t}$. Thus, the overall STDs of the filtered datasets were dominated by the STDs in tissue. (3) Both $\sigma$ and $\sigma_{t}$ were reduced as the filter size was increased. (4) The mean intensity in the datasets $\left(I_{m}\right)$ was near zero in brain tissue, air, and the entire imaging volume. For example, the mean of the filtered dataset with iH $32 \times 24$ was less than $0.01 \%$ of the maximum intensity of the original dataset. (5) Both $\sigma$ and $\sigma_{t}$ of an iH filtered dataset were higher than that of an $\mathrm{iF}$ filtered dataset with the same filter size.

The percentage of voxels excluded by the second condition in (3), as shown in a function of $\eta$ in Figure 1, was less than $0.026 \%$ when $\eta \geq 6$. Therefore, $\eta=6$ was selected in this study because it only excludes a very small portion of voxels.

Figure 2 shows the MIP of the STD-scaled BS-MRV datasets, $I_{\mathrm{BS}-\mathrm{MRV}}(x, y, z)$, processed with the $\mathrm{iH}(\mathrm{a})$ and $\mathrm{iF}$ (b) filters at different filter sizes. The venous vasculature was well depicted in these images. The visibility of the veins in 


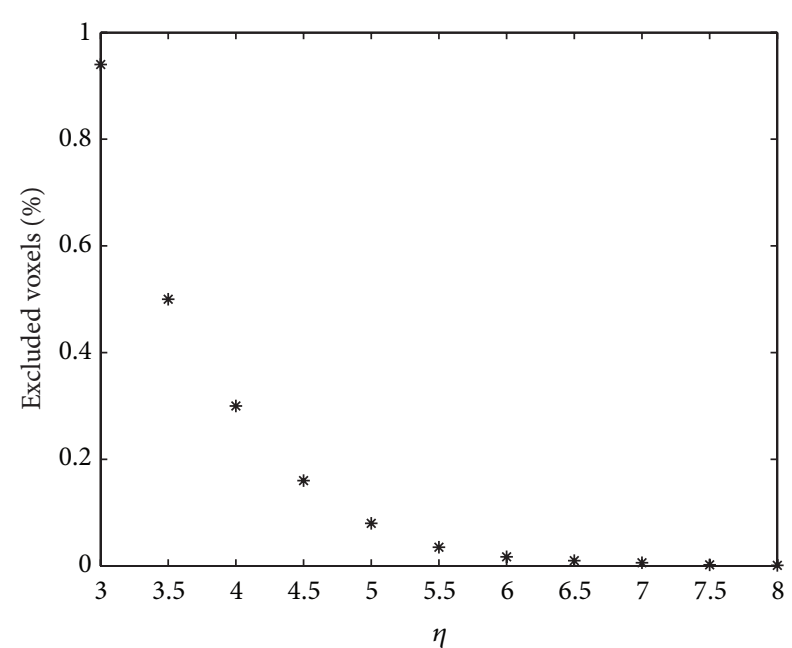

FIgURE 1: This figure shows the percentage of voxels excluded by the second condition in (3) as a function of $\eta$. The excluded voxels were only $0.026 \%$ when $\eta=6$.

the images processed with an iF filter was comparable with that in the images processed with an $\mathrm{iH}$ filter. It was observed that the filter size only had a minor effect on the overall image quality. The shade in the peripheral regions of the brain, as indicated by the thick short arrows, and at the third ventricle, as indicated by the thin short arrows, was more conspicuous with smaller filter sizes. The BS-MRV datasets at the middle two columns appear to have similar vessel visibility compared to the other images. Although not shown here, the BS-MRV datasets processed with larger filter sizes than the ones shown in this figure demonstrated gradually increased noise level and decreased venous contrast.

Figure 3 shows the $512 \times 384 \times 50$ (interpolated to $1024 \times$ $768 \times 100$ ) dataset, the single slice (top row), and mIP (bottom row) of the magnitude images (a and e), MRV obtained by applying the conventional SWI postprocessing algorithm (homodyne filter size $64 \times 48$ ) (b and $\mathrm{f}$ ), conventional SWI with a median filter $(5 \times 5 \times 5)(\mathrm{c}$ and $\mathrm{g})$, and BS-MRV technique with iH filtering $\left(2 H_{x} \times 2 H_{y}=32 \times 24\right)(\mathrm{d}$ and h). Severe off-resonance artifacts (as indicated by the bright arrows) and signal loss at the peripheral regions of the brain were observed in (b) and (f). Slight off-resonance artifacts (as indicated by the arrows) were also observed in (c) and ( $\mathrm{g}$ ). The visibility of larger veins was reduced in (c) and (g). In contrast, the BS-MRV images in (d) and (h) show good visibility of the venous vasculature, effective background suppression, and the absence of off-resonance artifacts. The veins adjacent to the regions of low signal intensity, such as around the globus pallidus, were largely obscured in the MIP of the magnitude data and SWI. But they were well depicted in the BS-MRV images.

The results of applying the BS-MRV technique to a 4echo MRAV dataset are shown in Figure 4. The data of Echo $2(\mathrm{TE}=19.7 \mathrm{~ms})$, Echo $3(\mathrm{TE}=24.8 \mathrm{~ms})$, and Echo $4(\mathrm{TE}=$ $39.0 \mathrm{~ms}$ ) are shown in the left, middle, and right column, respectively. The $\mathrm{mIP}$ images of original $3 \mathrm{D}$ magnitude data are shown at the top row. The MIP images of the 3D MRV processed by applying the conventional SWI technique to the $3 \mathrm{D}$ complex data are shown in the middle row. The bottom row shows the MIP images of the 3D data obtained by applying the BS-MRV technique to the same 3D magnitude data as shown in the top row. The off-resonance artifact introduced by the phase distortion in the frontal region with severe field inhomogeneity in the SWI images, as indicated by an arrow, was absent in the BS-MRV data. The regions with high level of iron deposits, such as the globus pallidus regions, indicated by the dashed circles, did not obscure the visibility of veins in the BS-MRV data either. The veins in the peripheral regions of the brain were obscured in the MIP display of the magnitude data and SWI data but were well depicted in the MIP of the BS-MRV data. In most of the brain regions, the visibility of the veins in the BS-MRV data was much better than that in the magnitude data and was comparable to that in the SWI data, especially at a shorter TE. The results demonstrate that the BS-MRV algorithm can provide adequate venous visibility in a relatively wide range of TE, even in regions of severe field inhomogeneity.

The BS-MRV algorithm can be readily applied to $3 \mathrm{D}$ SPGR acquired with a phased-array coil. The composite images were constructed by the squared sum of the 8 data sets acquired from each of the coil elements. Figure 5 shows the mIPs of magnitude images (top row) and MRV data in a subvolume of 18 slices obtained by applying the BS-MRV algorithm to the $3 \mathrm{D}$ composite images in five subjects.

\section{Discussion}

Background suppression is a commonly used approach in MR vascular imaging $[15,25,26]$. In TOF MRA, background signal is saturated by repeated RF excitations in the imaging volume. The contrast of vessels can be further enhanced by the additional saturation of brain tissue using a magnetization transfer technique, in which off-resonance RF pulses with a relatively high power are repeatedly applied [27]. Median filters have been applied in the image domain for the suppression of background tissue in angiography [25] and BOLD venography [26]. In such spatial filtering approaches, the selection of the kernel size of a median filter will affect the visibility of the veins when the size of the kernel is comparable with the size of the veins, as shown in Figures 3(c) and 3(g). As an extension to these approaches of background suppression, the BS-MRV technique was developed to enhance the visibility of small veins through the digital suppression of the background signal by applying HP filters in the Fourier domain in postprocessing. The results shown in this study indicate that background suppression achieved by using a Fourier domain HP filter can effectively enhance the visibility of small veins when the size of the HP filter is in a relatively wide range.

In SWI, the contrast of the veins is obtained primarily from the susceptibility induced phase shift. This phase shift is enhanced in the phase mask by applying an HP filter in the Fourier domain to suppress the background phase. The contrast of the veins is further enhanced by multiplying the phase mask to the magnitude images several times. 


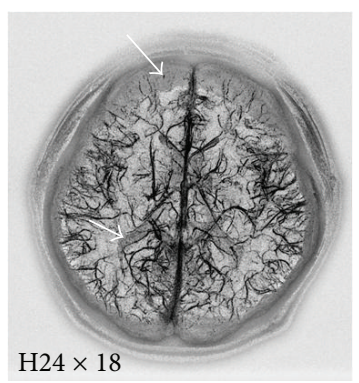

$\mathrm{H} 24 \times 18$

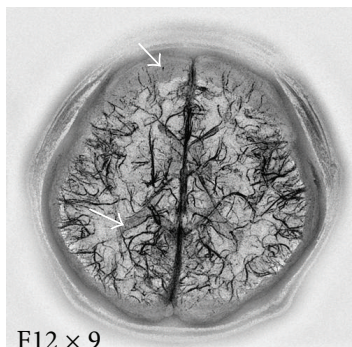

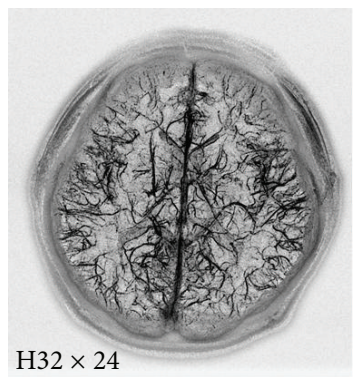

$\mathrm{H} 32 \times 24$

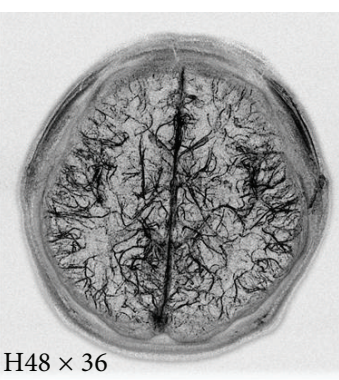

(a)

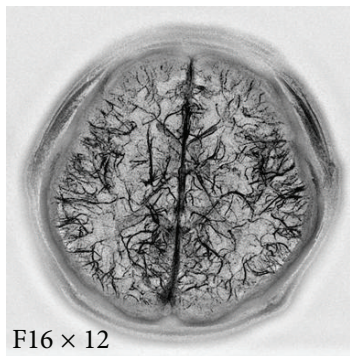

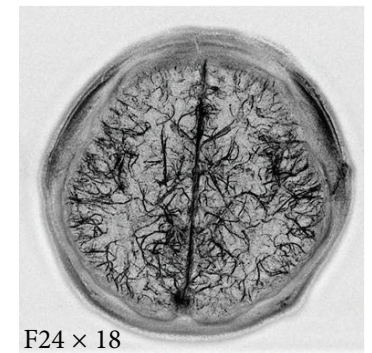

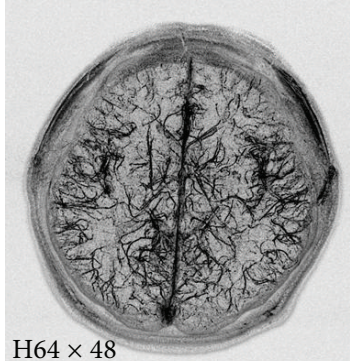

$\mathrm{H} 64 \times 48$

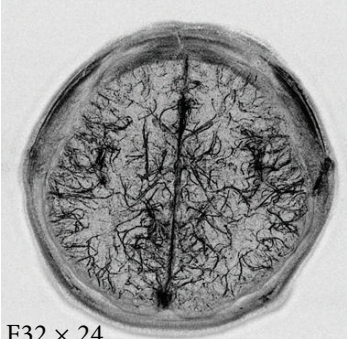

(b)

FIgURE 2: The mIP of the STD-scaled BS-MRV $(512 \times 384 \times 50$, interpolated to $1024 \times 768 \times 100)$ processed with the iH (top row) and iF (bottom row) filters at different filter sizes. At the top row, the numbers following the label "iH" indicate the filter size $2 H_{x} \times 2 H_{y}$. At the bottom row, the numbers following the label " $\mathrm{iF}$ " indicate the filter size $2 R_{x} \times 2 R_{y}$. In some of the peripheral regions of the brain, as indicated by thick long arrows, the visibility of the veins was obscured by the scalp.

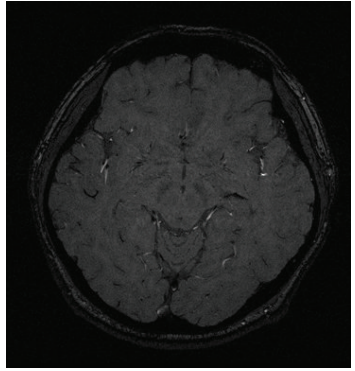

(a)

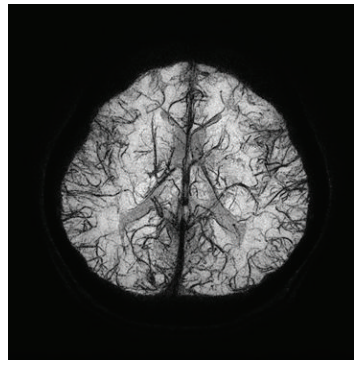

(e)

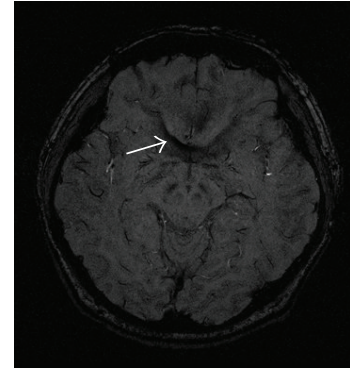

(b)

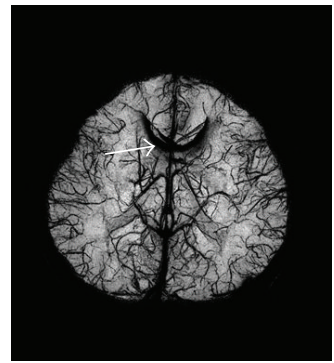

(f)

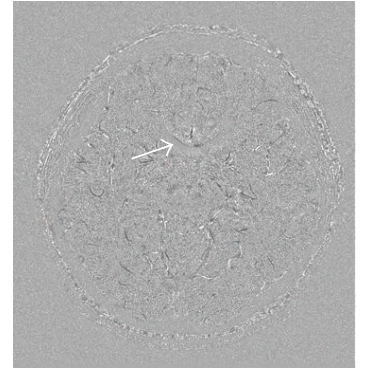

(c)

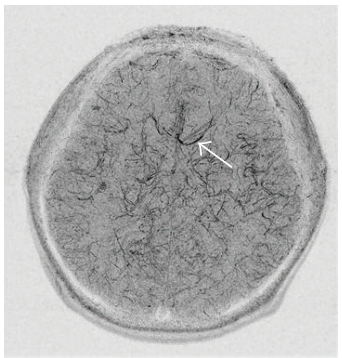

(g)

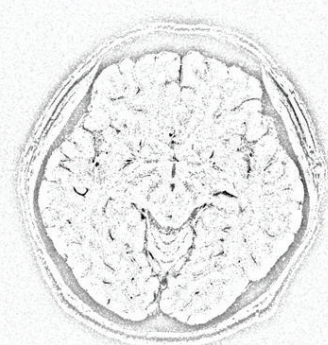

(d)

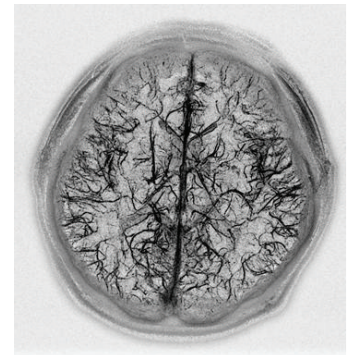

(h)

Figure 3: This figure shows the $512 \times 384 \times 50$ (interpolated to $1024 \times 768 \times 100$ ) dataset, the single slice (top row), and mIP (bottom row) of the magnitude images (a and e), MRV obtained by applying the conventional SWI postprocessing algorithm (homodyne filter size $64 \times 48$ ) $(\mathrm{b}$ and $\mathrm{f})$, conventional SWI with a median filter $(5 \times 5 \times 5)\left(\mathrm{c}\right.$ and g), and BS-MRV technique with iH filtering $\left(2 \mathrm{H}_{x} \times 2 \mathrm{H}_{y}=32 \times 24\right)(\mathrm{d}$ and h). Severe off-resonance artifact (as indicated by bright arrows) and signal loss at the peripheral regions of the brain are observed in (b) and (f). Slight off-resonance artifacts (as indicated by arrows) were also observed in (c) and (g). 

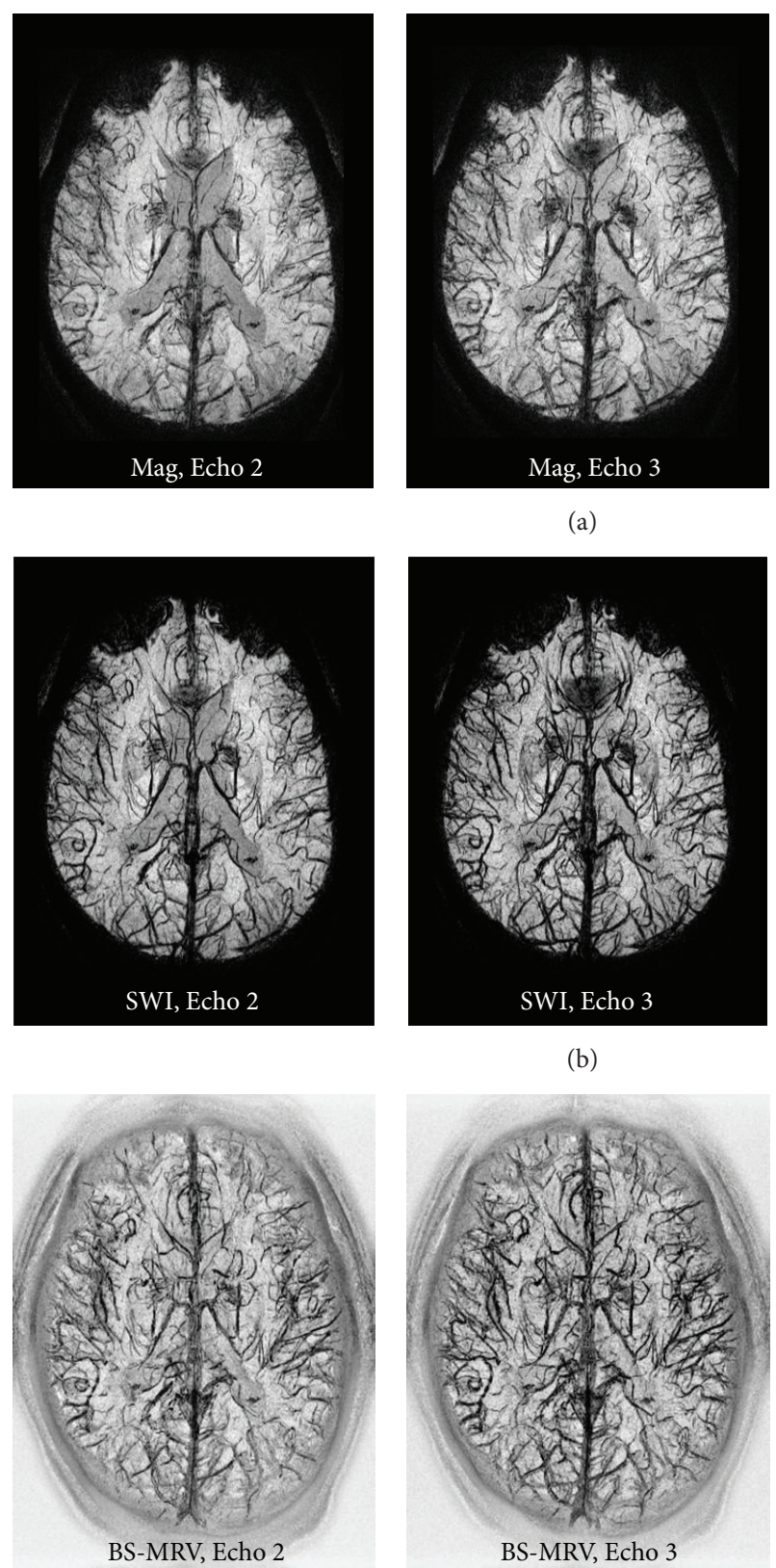

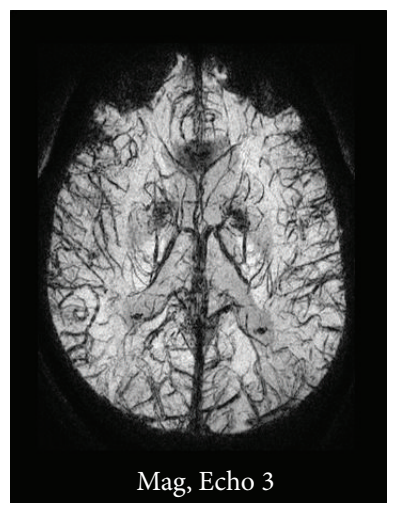

(a)

(b)
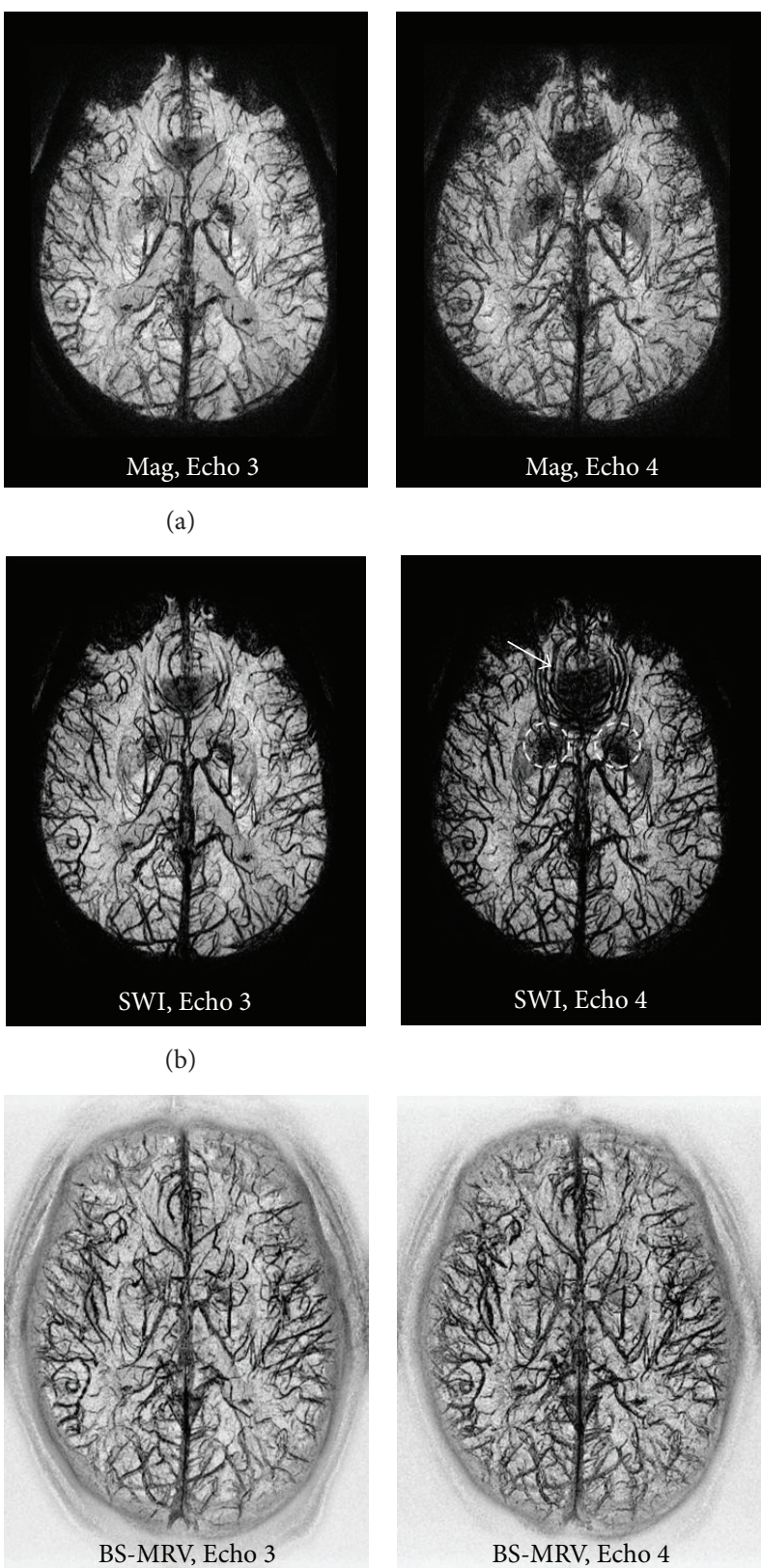

(c)

FIGURE 4: This figure shows the comparison of the magnitude data, SWI data, and BS-MRV data acquired with a 4-echo acquisition technique at Echo $2(\mathrm{TE}=19.7 \mathrm{~ms}$, left column), Echo $3(\mathrm{TE}=24.8 \mathrm{~ms}$, middle column), and Echo $4(\mathrm{TE}=39.0 \mathrm{~ms}$, right column). This figure also shows the comparison of the original 3D magnitude (top row), the conventional SWI (middle row), and the BS-MRV (bottom row) using the same datasets. The off-resonance artifact in the frontal region with severe field inhomogeneity in SWI, as indicated by an arrow, was absent in BS-MRV. The regions with high level of iron deposits, such as the globus pallidus regions indicated by the dashed circles, did not obscure the visibility of veins in the BS-MRV.

Conversely, only the magnitude data are used in the BS-MRV technique. It is noteworthy that the difference of susceptibility phase between venous blood and parenchyma within a voxel causes partial-volume signal cancellation in the magnitude data. This, therefore, has a substantial effect on the contrast of veins in BS-MRV, despite not using the phase of the acquired data in the technique. The results of this study demonstrate that MRV with good visibility of small veins can be obtained by suppressing the background signal in the magnitude data without using the phase data. Suppressing the background signal in postprocessing is an effective approach for the proper display of the venous contrast available in the magnitude data.

The use of magnitude images alone for MRV has a major practical advantage. On most of the clinical MRI scanners, phase data are not readily accessible to users. The BR-MRV 


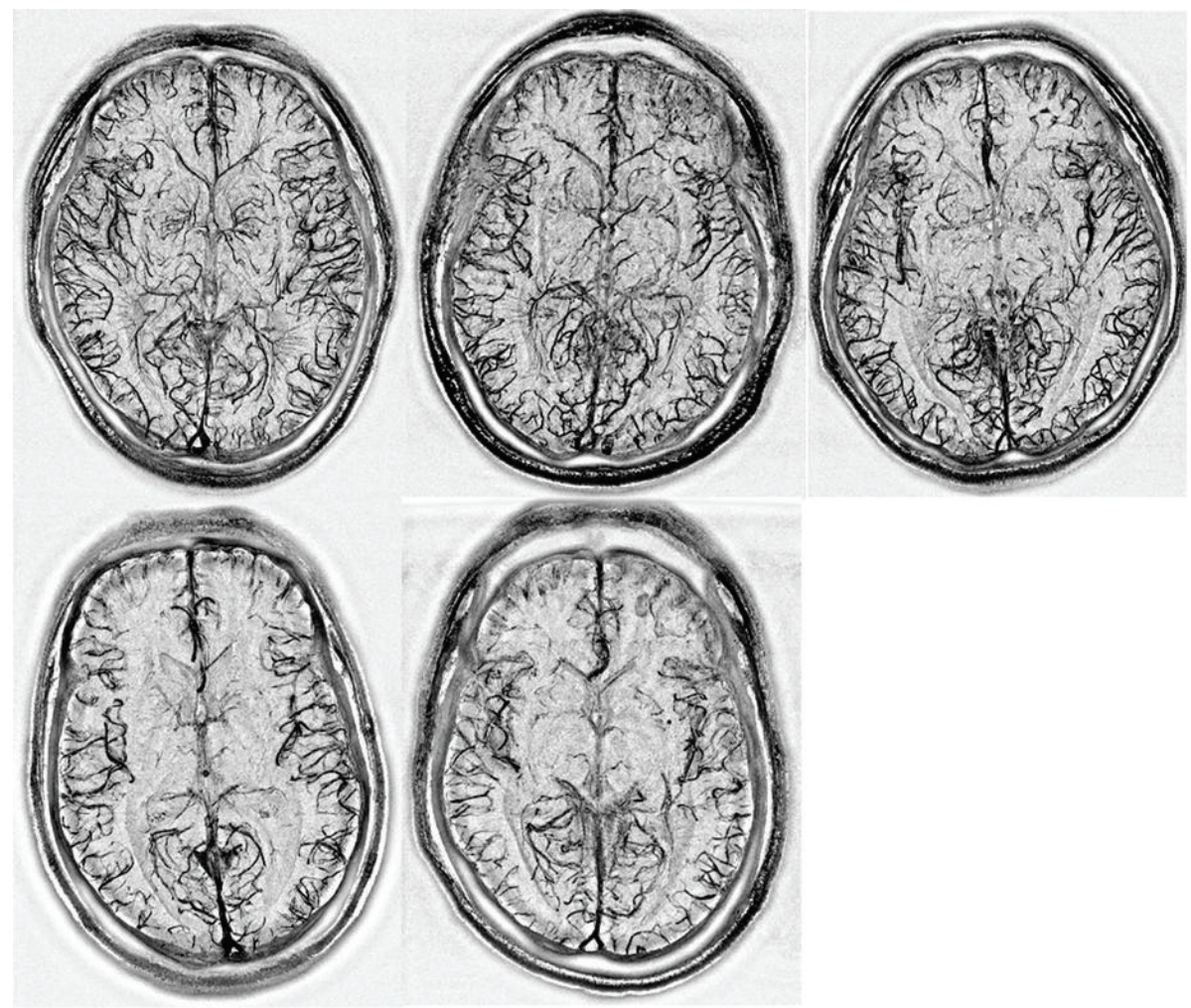

FIGURE 5: This figure shows the mIP of BS-MRV data of 5 subjects in a subvolume of 18 slices acquired with 8-channel phased-array RF coils.

technique can be directly applied to the magnitude images reconstructed on the scanners, including the images previously acquired. This simplicity of the BS-MRV technique overcomes a major obstacle for routine MRV applications in the clinical environment.

The off-resonance effect causes major artifacts in SWI by introducing phase wraps in regions with severe field inhomogeneity. The off-resonance artifact, commonly appearing as dark bands in the MIP display of SWI, can obscure the visibility of veins in the frontal and temporal regions of the brain. The off-resonance artifact can also appear as vein-like dark lines and be mistaken for veins. However, BS-MRV is not subject to the off-resonance artifact because it does not use the phase data.

Signal loss observed in the peripheral regions of the brain in the MIP display of SWI was substantially reduced in BS-MRV. The signal reduction in the magnitude images in iron-rich regions, such as globus pallidus, can obscure the visibility of the adjacent veins in the MIP display of the magnitude or SWI data. In addition, CSF often has a lower intensity than brain tissue, due to more severe T1 saturation in CSF. The third ventricle often appears darker than the other brain regions and, therefore, reduces the contrast of the veins along the path of its projection. Using the background suppression approach, the adverse effects of such a decrease of background signal on the visibility of veins in the mIP projection can be effectively reduced.

The contrast of veins and the severity of the off-resonance artifacts are strongly dependent on the filter size in SWI [17].
With the use of STD scaling, the contrast of veins in the BSMRV data remains more or less the same with a wide range of filter sizes. Because of this, the adequate display of the BS-MRV data becomes more feasible with minimal operator interaction. It was further noted that both $\mathrm{iH}$ and iF filters provide comparable results at the same filter size.

The BS-MRV technique can be directly applied to the composite magnitude images acquired with phased-array coils, resulting in a substantial simplification of the data processing compared to the conventional SWI technique. In the conventional approach for combining data acquired with multiple coils, only magnitude data from these coils are used to form composite images with a squared-sum algorithm. This approach is not adequate in SWI, because of the loss of phase information in the composite images. For SWI, phase shift in the individual dataset acquired at each coil must be preserved in the composite images [26]. Any coildependent phase offset has to be properly addressed. These technical issues in multicoil acquisitions of SWI are no longer problematic in BS-MRV.

There are a few weaknesses and potential pitfalls with the BS-MRV technique. Unlike the SWI technique, the scalp signal appears dark in the BS-MRV data and can obscure the visibility of veins on the surface of the brain. In addition, the signal of major veins, such as the sagittal sinus, can be suppressed because of the use of the HP filters. For clinical diagnoses of vascular diseases of the major veins, it would be preferable to use the original magnitude images. 


\section{Conclusions}

Good visualization of the cerebral venous vasculature can be obtained by applying proper background suppression to the magnitude of a 3D flow-compensated SPGR dataset, acquired with a relatively wide range of TE, through postprocessing. The proposed BS-MRV technique can provide good visualization of the cerebral venous vasculature even in regions with severe field inhomogeneity. With the effective background suppression, the veins adjacent to the regions of low signal intensity, such as globus pallidus, can be well depicted. Without using the phase data, the implementation of the proposed postprocessing technique on clinical scanners can be substantially simplified.

\section{Conflict of Interests}

The authors declare that there is no conflict of interests regarding the publication of this paper.

\section{Acknowledgments}

This work was partially supported by the National Key Basic Research Program of China (2013CB329501), the National Natural Science Foundation of China (81371518, 81261120411, 61372024, 81271530, and 81171310), and the Key Projects in the National Science \& Technology Pillar Program during the Twelfth Five-Year Plan Period (2011BAI12B01, 2012BAI10B04). The authors are grateful to Dr. Lynn Ho for editing the paper.

\section{References}

[1] H. P. Mattle, K. U. Wentz, R. R. Edelman et al., "Cerebral venography with MR,” Radiology, vol. 178, no. 2, pp. 453-458, 1991.

[2] L. Liauw, M. A. van Buchem, A. Spilt et al., "MR angiography of the intracranial venous system," Radiology, vol. 214, no. 3, pp. 678-682, 2000.

[3] M. H. Pui, "Cerebral MR venography," Clinical Imaging, vol. 28, no. 2, pp. 85-89, 2004.

[4] N. Rollins, C. Ison, T. Reyes, and J. Chia, "Cerebral MR venography in children: comparison of $2 \mathrm{D}$ time-of-flight and gadolinium-enhanced 3D gradient-echo techniques," Radiology, vol. 235, no. 3, pp. 1011-1017, 2005.

[5] K.-O. Lövblad, J. Schneider, C. Bassetti et al., "Fast contrastenhanced MR whole-brain venography," Neuroradiology, vol. 44, no. 8, pp. 681-688, 2002.

[6] K. Kirchhof, T. Welzel, O. Jansen, and K. Sartor, "More reliable noninvasive visualization of the cerebral veins and dural sinuses: comparison of three MR angiographic techniques," Radiology, vol. 224, no. 3, pp. 804-810, 2002.

[7] A. Tomasian, N. Salamon, M. S. Krishnam, J. P. Finn, and J. P. Villablanca, "3D high-spatial-resolution cerebral MR venography at 3T: a contrast-dose-reduction study," The American Journal of Neuroradiology, vol. 30, no. 2, pp. 349-355, 2009.

[8] J. R. Reichenbach, R. Venkatesan, D. J. Schillinger, D. K. Kido, and E. M. Haacke, "Small vessels in the human brain: MR venography with deoxyhemoglobin as an intrinsic contrast agent," Radiology, vol. 204, no. 1, pp. 272-277, 1997.
[9] J. R. Reichenbach, L. Jonetz-Mentzel, C. Fitzek et al., "Highresolution blood oxygen-level dependent $\mathrm{MR}$ venography (HRBV): a new technique," Neuroradiology, vol. 43, no. 5, pp. 364-369, 2001.

[10] E. M. Haacke, Y. Xu, Y.-C. N. Cheng, and J. R. Reichenbach, "Susceptibility weighted imaging (SWI)," Magnetic Resonance in Medicine, vol. 52, no. 3, pp. 612-618, 2004.

[11] V. Sehgal, Z. Delproposto, E. M. Haacke et al., "Clinical applications of neuroimaging with susceptibility-weighted imaging," Journal of Magnetic Resonance Imaging, vol. 22, no. 4, pp. 439450, 2005.

[12] A. Deistung, A. Rauscher, J. Sedlacik, J. Stadler, S. Witoszynskyj, and J. R. Reichenbach, "Susceptibility weighted imaging at ultra high magnetic field strengths: theoretical considerations and experimental results," Magnetic Resonance in Medicine, vol. 60, no. 5, pp. 1155-1168, 2008.

[13] A. Rauscher, J. Sedlacik, M. Barth, E. M. Haacke, and J. R. Reichenbach, "Nonnvasive assessment of vascular architecture and function during modulated blood oxygenation using susceptibility weighted magnetic resonance imaging," Magnetic Resonance in Medicine, vol. 54, no. 1, pp. 87-95, 2005.

[14] A. Rauscher, M. Barth, J. R. Reichenbach, R. Stollberger, and E. Moser, "Automated unwrapping of MR phase images applied to BOLD MR-venography at 3 Tesla," Journal of Magnetic Resonance Imaging, vol. 18, no. 2, pp. 175-180, 2003.

[15] A. Rauscher, M. Barth, K.-H. Herrmann, S. Witoszynskyj, A. Deistung, and J. R. Reichenbach, "Improved elimination of phase effects from background field inhomogeneities for susceptibility weighted imaging at high magnetic field strengths," Magnetic Resonance Imaging, vol. 26, no. 8, pp. 1145-1151, 2008.

[16] C. Denk and A. Rauscher, "Susceptibility weighted imaging with multiple echoes," Journal of Magnetic Resonance Imaging, vol. 31, no. 1, pp. 185-191, 2010.

[17] Z. Jin, L. Xia, and Y. P. Du, "Reduction of artifacts in susceptibility-weighted MR venography of the brain," Journal of Magnetic Resonance Imaging, vol. 28, no. 2, pp. 327-333, 2008.

[18] J. Neelavalli, Y.-C. N. Cheng, J. Jiang, and E. M. Haacke, "Removing background phase variations in susceptibilityweighted imaging using a fast, forward-field calculation," Journal of Magnetic Resonance Imaging, vol. 29, no. 4, pp. 937-948, 2009.

[19] D. S. J. Pandian, C. Ciulla, E. M. Haacke, J. Jiang, and M. Ayaz, "Complex threshold method for identifying pixels that contain predominantly noise in magnetic resonance images," Journal of Magnetic Resonance Imaging, vol. 28, no. 3, pp. 727-735, 2008.

[20] Y. P. Du and Z. Jin, "Robust tissue-air volume segmentation of MR images based on the statistics of phase and magnitude: its applications in the display of susceptibility-weighted imaging of the brain," Journal of Magnetic Resonance Imaging, vol. 30, no. 4, pp. 722-731, 2009.

[21] Z. Jin, L. Xia, and Y. P. Du, "Semi-automated volume segmentation of the brain based on a level set approach," in Proceedings of the International Society for Magnetic Resonance in Medicine (ISMRM '09), p. 2870, Honolulu, Hawaii, USA, 2009.

[22] M. J. Lowe and J. A. Sorenson, "Spatially filtering functional magnetic resonance imaging data," Magnetic Resonance in Medicine, vol. 37, no. 5, pp. 723-729, 1997.

[23] Y. P. Du, Z. Jin, Y. Hu, and J. Tanabe, "Multi-echo acquisition of MR angiography and venography of the brain at 3 Tesla," Journal of Magnetic Resonance Imaging, vol. 30, no. 2, pp. 449-454, 2009. 
[24] E. M. Haacke, E. D. Lindskogj, and W. Lin, "A fast, iterative, partial-fourier technique capable of local phase recovery," Journal of Magnetic Resonance, vol. 92, no. 1, pp. 126-145, 1991.

[25] A. L. Alexander, B. E. Chapman, J. S. Tsuruda, and D. L. Parker, "A median filter for 3D FAST spin echo black blood images of cerebral vessels," Magnetic Resonance in Medicine, vol. 43, no. 2 , pp. 310-313, 2000.

[26] E. G. Kholmovski and D. L. Parker, "High resolution magnetic resonance venography at 3 Tesla: optimized acquisition, reconstruction, and post-processing," in Proceedings of the International Society for Magnetic Resonance in Medicine (ISMRM '06), p. 810, Seattle, Wash, USA, 2006.

[27] S. D. Wolff, J. Eng, and R. S. Balaban, "Magnetization transfer contrast: method for improving contrast in gradient-recalledecho images," Radiology, vol. 179, no. 1, pp. 133-137, 1991. 


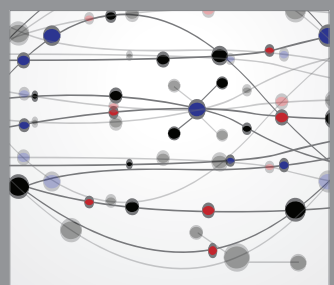

The Scientific World Journal
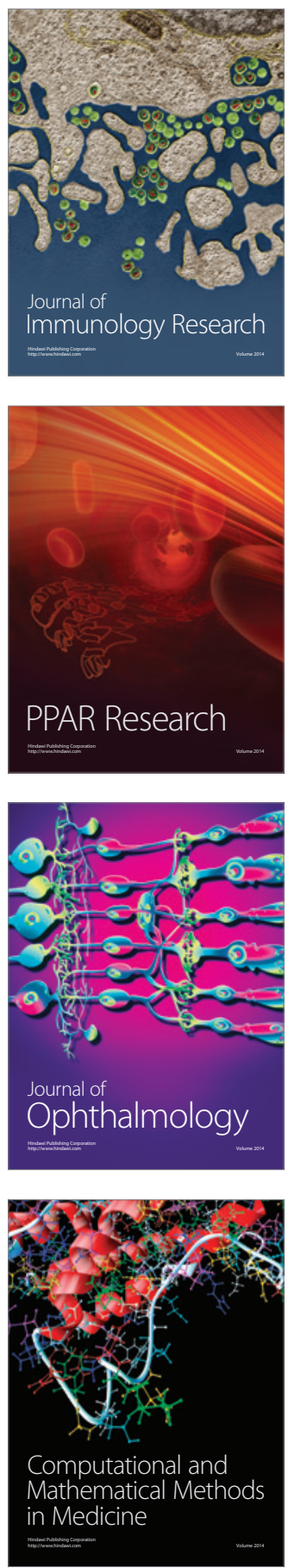

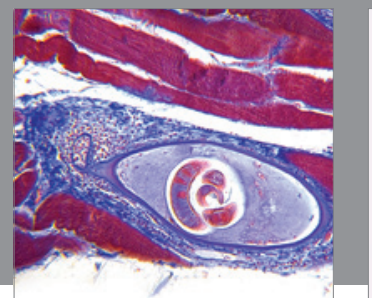

Gastroenterology

Research and Practice
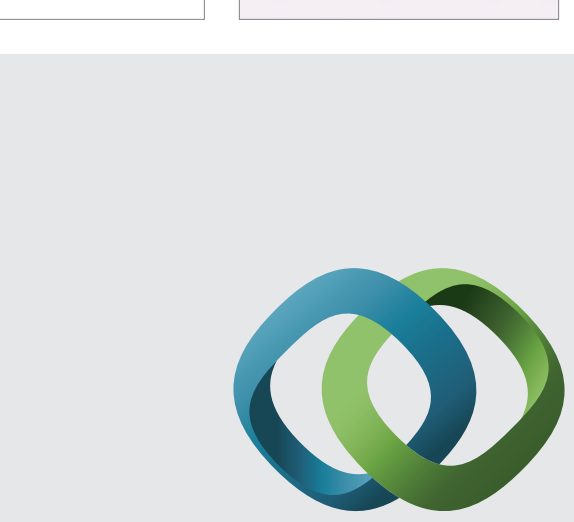

\section{Hindawi}

Submit your manuscripts at

http://www.hindawi.com
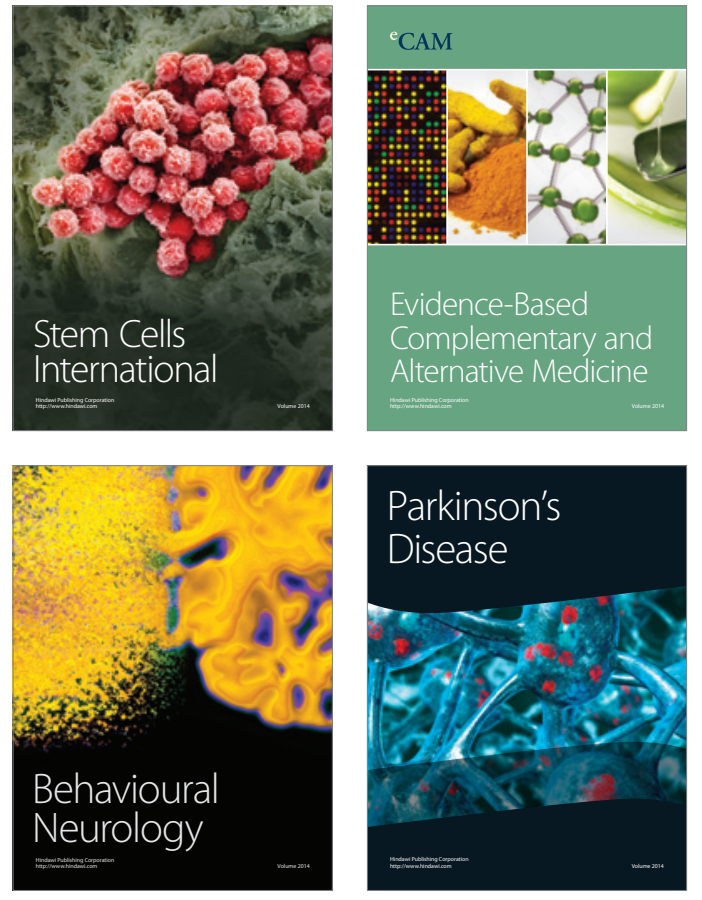
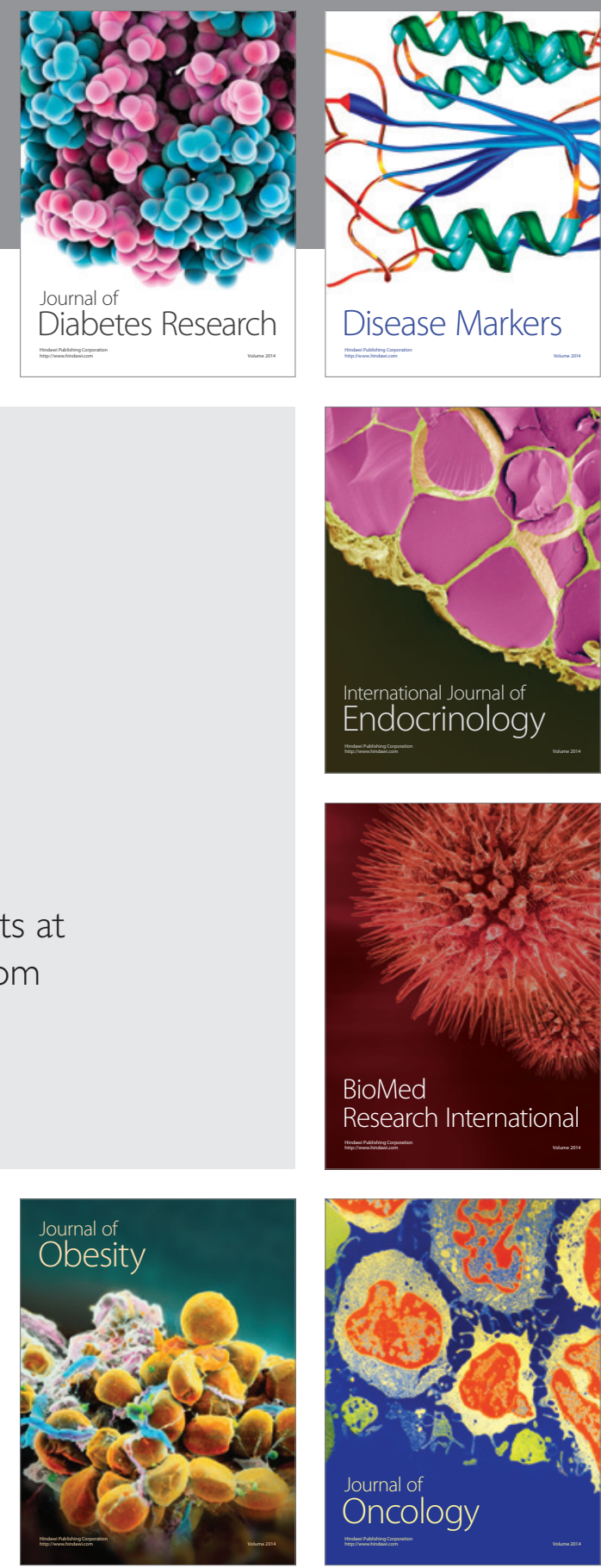

Disease Markers
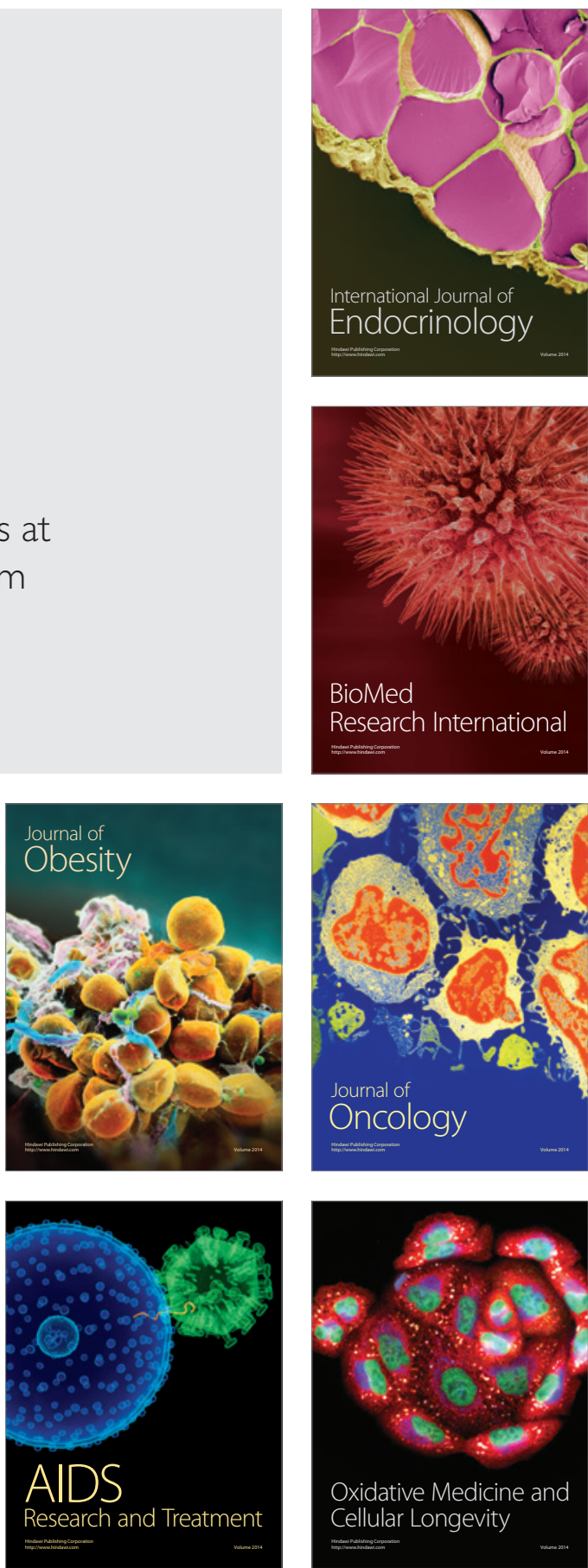